\title{
Debates: ¿Podemos manejar sustentablemente el bosque nativo de Patagonia Norte?
}

\author{
Javier Grosfeld ${ }^{1, \infty}$; Luis Chauchard² \& Juan H. Gowda ${ }^{3}$ \\ ${ }^{1}$ CONICET CCT Patagonia Norte, CRUB-Universidad Nacional del Comahue, San Carlos de Bariloche, Argentina. \\ ${ }^{2}$ Universidad Nacional del Comahue, Administración de Parques Nacionales, San Martín de los Andes, Neuquén, Argentina. \\ ${ }^{3}$ INIBIOMA (CONICET-Universidad Nacional del Comahue), San Carlos de Bariloche, Argentina.
}

\begin{abstract}
Resumen. Los bosques del norte de la Patagonia tienen una larga historia de uso, siendo uno de los ecosistemas forestales más estudiados de Argentina. Sin embargo, existen pocos ejemplos de bosques nativos bajo manejo sustentable. Proponemos que esto se debe principalmente a la falta de voluntad de las autoridades de aplicación, organismos de gestión, investigadores y técnicos del sector por asistir a productores forestales en la implementación de la legislación vigente. Históricamente, los productores han sido ignorados por un sistema administrativo que no ha buscado adaptarse a sus necesidades, y por un sistema científico-tecnológico que no ha utilizado sus conocimientos empíricos para generar propuestas realistas de manejo. Consideramos que, si bien el manejo sustentable de los bosques patagónicos pareciera ser técnicamente viable, su implementación dependerá de la voluntad del sector público por generar las condiciones para un diálogo intersectorial que integre distintos tipos de saberes.
\end{abstract}

[Palabras clave: gestión forestal, confianza, productores, sistema científico-tecnológico, uso del suelo]

\begin{abstract}
Aвstract. Can we sustainably manage the native forest of North Patagonia? The native forests of Northern Patagonia have a long history of human use, being one of the most studied ecosystems of Argentina. However, there are few examples of sustainably managed native forests. We propose that this is mainly due to the lack of political will from regional authorities, forest administration agencies, technicians and researchers in assisting forest owners to implement current legislation. Historically, forest owners have been ignored by an administrative system that has not considered their needs as well as by a scientific-technologic system that not used their empirical knowledge to develop realistic management packages. We consider that, even though the sustainable management of Patagonian native forests seems to be technically feasible, its implementation will depend on the will of the public sector to generate better conditions for an intersectoral collaboration that integrates different kinds of knowledge.
\end{abstract}

[Keywords: forest administration, trust, producers, R\&D, land use]

\section{LA CIENCIA Y LA TÉCNICA, VARIADOS MATICES DEL MANEJO SUSTENTABLE}

Para responder esta pregunta es necesario analizar el concepto de manejo sustentable en varias dimensiones. Empecemos por la básica, la técnica, y luego avanzaremos sobre aspectos institucionales. El manejo de cualquier recurso tiene implícito un propósito, su uso, ya sea para generar bienes tangibles o servicios intangibles. El principio que debe regir para alcanzar este propósito es que los sistemas y servicios perduren (persistencia de la productividad del sistema). En el caso de los bosques nativos podríamos definir tres grandes ejes de actuación: conservar, producir y restaurar. En los tres casos, al bosque se le asigna un valor que se debe mantener a perpetuidad. En el sentido más estricto, la conservación postula como objetivo principal del manejo mantener a perpetuidad el ecosistema boscoso en su estado actual. El manejo productivo plantea como objetivo

Editora invitada: Rosina Soler $\bar{\triangle}$ grosfeldje@comahue-conicet.gob.ar obtener una renta del objeto manejado, sea puntual, oportunista o sostenida. La restauración, por último, establece como principal objetivo recuperar un valor perdido (Figura 1). Vale aclarar que la restauración puede estar orientada a recuperar un valor de conservación o de producción perdido. Manejar implica enfocar esfuerzos y recursos hacia un determinado objetivo. Tanto la acción como la inacción pueden surgir de una decisión de manejo. Toda decisión de manejo implica un cambio en el valor del objeto manejado, cuya dirección será gatillada por el paradigma en que vivimos y su resultado será contextual.

El concepto "sustentable" de un proceso se hace más complejo cuanto más se acerca a la gestión, pues explicita que ésta se debe realizar integrando los múltiples factores que están afectados o involucrados. Si bien es difícil definir un período adecuado para asociar el manejo forestal como un proceso

Recibido: 7 de Junio de 2018

Aceptado: 4 de Diciembre de 2018 


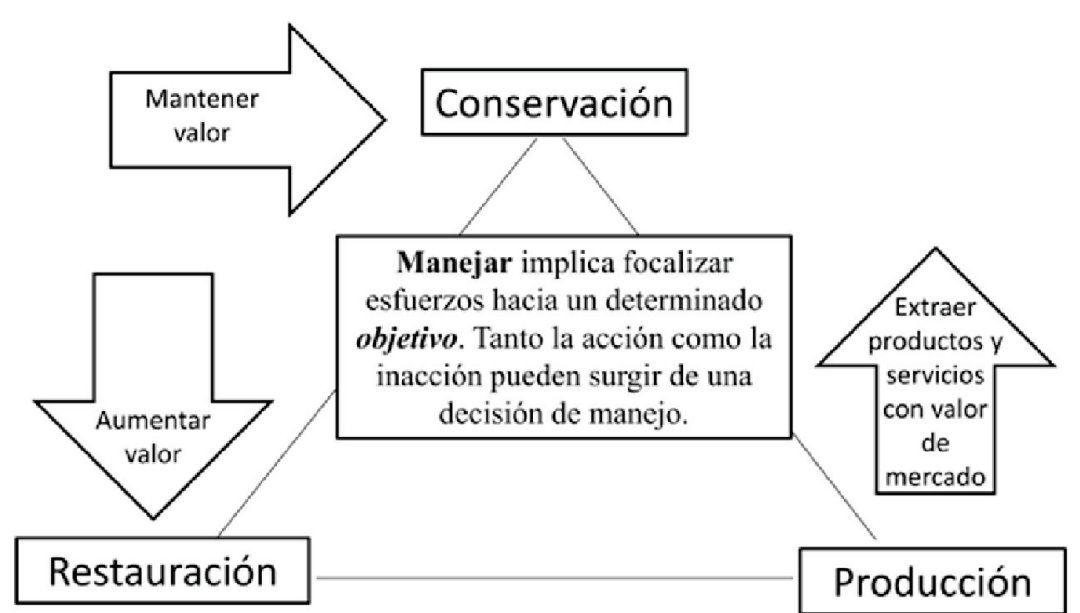

Figura 1. Esquema simple de tres dimensiones del manejo forestal y su relación con el valor que le asignamos en relación con su existencia, funciones, biodiversidad, servicios o productos.

Figure 1. Simple scheme of three dimensions of forest management and its relation to the value we assign according to several criteria such as its stocks, functions, biodiversity, services and/or products. sustentable, es fundamental tener al menos una visión de mediano plazo para alcanzar metas claras acordadas que permitan hacer una valoración objetiva una vez transcurrido dicho período. Un manejo sustentable, que implica perpetuidad de dos elementos indivisibles sistema boscoso y sociedad, sería sólo una expresión de deseos si no considera sus efectos en un horizonte de largo plazo, relacionado con la dinámica natural y con la provisión de bienes y servicios de los bosques nativos, y con los impactos económicos, ecológicos y sociales de corto y mediano plazo que el manejo generará. Esto se hace más acuciante en el escenario actual de cambio climático y los manejos adaptativos necesarios para disminuir la vulnerabilidad de los ecosistemas forestales, ya sea para la conservación estricta como para la producción.

Desde fines de la década del 80, la aplicación del término sustentabilidad a toda acción del ser humano implica asociar e integrar los factores económicos, sociales y ambientales. Sin embargo, la sustentabilidad del manejo continuará dependiendo del resultado económico para quien lo ejecute y para quienes están vinculados de forma directa con la cadena productiva o conglomerado (cluster). Todo manejo implica, por lo tanto, una valoración del sistema que puede (o no) estar asociada al precio de los productos y servicios que resulten de dicho manejo en un mercado determinado, presente o futuro. A la hora de definir un manejo adecuado para el bosque nativo debemos no sólo llegar a un acuerdo como sector y sociedad sobre si buscamos mantener, aumentar o extraer valor del bosque, sino también sobre cómo definimos el valor de los diversos servicios y productos asociados con el mismo, y si debemos retribuir económicamente a quien lo gestiona. Planificar a diferentes escalas y buscar acuerdos para componer los objetivos del manejo es un tema central al delinear una estrategia eficaz y aplicable. Para ello, un paso clave es establecer quiénes son los actores o interlocutores directa o indirectamente involucrados para la construcción de acuerdos sobre dichos objetivos del manejo. En el uso de los recursos forestales, lo que es socialmente aceptable (como veremos más adelante) cambia a lo largo de nuestra historia. La valoración ambiental del manejo forestal también fue evolucionando y haciéndose más compleja, quizás en mayor medida que la de los componentes social y económico. Como ecosistema, el bosque representa la casa en donde gran parte del proceso inicial de la cadena de producción se lleva a cabo; allí se generan las interacciones con los otros dos componentes. Sin embargo, es importante tener en cuenta que analizar la sustentabilidad de un proceso aislando uno de los componentes y sin tener en cuenta las interacciones del resto, limita la validez de los resultados.

Así planteado, en la Argentina, el concepto de manejo forestal sustentable tiene una connotación académica fuerte. Desde el momento que se analiza la decisión de iniciar el manejo de un bosque, siguiendo con su ejecución o gestión y cerrando el momento productivo con la industrialización o comercialización, existen otros factores que determinan el éxito del proyecto, es decir su sustentabilidad. En efecto, la institucionalidad, representada por el Estado y su calidad de gestión, a través de las políticas públicas para el sector (también vistas integralmente y vinculadas con la producción, la conservación, 
el marco normativo, el régimen impositivo, la regulación del mercado, la seguridad e higiene, etc.) $y$, fundamentalmente, su capacidad de implementación de éstas, son condicionantes esenciales para un manejo que se pretende sustentable.

\section{DE LA ACADEMIA AL MANEJO "TÉCNICAMENTE" SUSTENTABLE}

A nivel mundial existe un gran conocimiento sobre el manejo de los bosques templados, con características similares a los nuestros, que sirve de inspiración y encuentra su correlato en estudios de las principales especies arbóreas de la región (e.g., Bava 1999; Bava and López Bernal 2005; Goldenberg et al. 2018; Rush and Sarasola 1999; Sola et al. 2015; Urretavizcaya 2006). En Patagonia Norte, los bosques se están recuperando luego de grandes incendios asociados a un paradigma de desarrollo agrícola y ganadero (Willis 1914). A pesar de que la ganadería sigue siendo la principal actividad económica de los pobladores y comunidades de nuestros bosques nativos, en algunas zonas de la región, la supresión de los fuegos y el cambio de uso de la tierra permitió que los bosques de coihue y de ciprés avancen sobre matorrales post-fuego y reduzcan las superficies históricamente pastoreadas o cultivadas; a la vez, esto indica el fuerte impacto de los factores históricos sobre estos ecosistemas (Gowda et al. 2012; Kitzberger and Veblen 1999).

En Patagonia Norte, con la excepción de los bosques hidrófilos que constituyen la denominada "Selva Valdiviana", la mayoría de las comunidades forestales están dominadas por especies pioneras. En términos generales, en aquellas regiones donde el fuego cumple un rol ecológico, los bosques pueden distinguirse en dos grandes grupos: aquellos con especies pirófobas (i.e., sensibles al fuego) y aquellos con especies pirófilas (i.e., adaptadas a los incendios [Kitzberger et al. 2016]). A la vez, se pueden encontrar algunas especies de características intermedias. Las especies pirófilas se recuperan de los incendios por rebrotes epicórmicos, como el pehuén (Araucaria araucana), el ñire (Nothofagus anctartica), el radal (Lomatia hirsuta), la laura (Schinus patagonicus), el raulí (Nothofagus alpina) y el roble pellín (Nothofagus obliqua) (Mundo et al. 2013; Raffaele et al. 2011; Tercero-Bucardo et al. 2007). Por su parte, ante un disturbio, las especies sensibles necesitan de la presencia de fuentes de semillas cercanas para su reproducción, como sucede en los ecosistemas dominados por el ciprés (Austrocedurs chilensis), el coihue (Nothofagus dombeyi) o la lenga (N. pumilio). La forma de reproducción determinaría la estrategia de manejo de cada tipo forestal, lo que permite transiciones de bosques pirófilos a pirofóbos si se facilita el ingreso de especies de mayor porte (Kitzberger et al. 2016). El uso actual de los bosques se relaciona con estas características, ya que los bosques pirófilos son los que suelen ser usados para la provisión de leña (Gowda et al. 2014). Desde el punto de vista teórico podemos proponer estrategias de manejo que permitirían asegurar tanto la persistencia de los bosques pirófilos y pirófobos, como de sus estados transicionales para responder a criterios de valoración impuestos por nuestra sociedad y que satisfagan las demandas locales y los condicionantes ecológicos (Blackhall et al. 2017). Sin embargo, a este conocimiento acumulado durante las últimas décadas se le suma un desafío mayor: considerar en los esquemas teóricos de manejo el impacto del cambio climático sobre los ecosistemas, fenómeno de resultados imprevisibles en el mediano y largo plazo. Este desafío, requiere usar los bosques bajo premisas de no aumentar su vulnerabilidad; por el contrario, se necesita mejorar su resistencia y, con ello, facilitar su resiliencia.

A diferencia de la mayoría de las regiones del país, en Patagonia Norte hay un cúmulo relevante de conocimiento en relación con la ecología y el manejo de los bosques nativos. Gran parte de los estudios orientados a la producción forestal está sintetizado en el Manual de Buenas Prácticas para el Manejo del Bosque Nativo (Chauchard et al. 2012), que encuentra su correlato en el Manual de Buenas Prácticas para Plantaciones Forestales en la Patagonia Andina (Chauchard et al. 2016). A pesar de que la superficie de bosque nativo con potencial de manejo productivo (que, según la clasificación dada por la Ley Nacional 26331, es muy superior a las 100000 hectáreas de forestaciones en las tres provincias de Patagonia Norte) se puede notar que la ciencia y la técnica tuvieron una atención y un avance diferencial entre ambos sistemas en la generación del conocimiento. Ello mismo se puede inferir a partir del número de páginas o el mismísimo "peso del saber" que cada manual posee (Manual de Bosque Nativo [122 páginas y 320 g] vs. Manual Plantaciones Forestales [531 páginas y 1920 g]). En gran medida, esto se debe a 
que la formación profesional académica y el ejercicio profesional posterior (ingenierías y tecnicaturas forestales) se enfocaron mayormente en la implantación y manejo de forestaciones con especies exóticas, situación que se vio favorecida por la existencia de un mecanismo fiscal de incentivo económico a los propietarios de las mismas. Inclusive, en el abordaje de las investigaciones o experimentaciones con bosques nativos, una gran proporción se centró en aspectos vinculados con la conservación de ecosistemas asociada a una visión de bosques casi prístinos o inalterables (biología, ecología o licenciaturas ambientales), y se relegan aquellos estudios que abordan los aspectos que definan los modelos productivos con base sustentable. Sin lugar a dudas, un plan estratégico regional para promover el manejo forestal sustentable debe incluir la articulación de los manejos de las forestaciones y de los bosques nativos.

Si bien hoy existe en la academia una base de conocimiento adecuada para comenzar a manejar nuestros bosques según criterios asociados con su estructura y dinámica, la mayoría de las prácticas de manejo no supera la escala experimental y son contados los casos de manejo a escala comercial. Un caso excepcional que se desarrolla en Patagonia Norte es el del manejo del bosque mixto de raulí, coihue y roble pellín en el Parque Nacional Lanín, único caso de manejo a escala productiva con 30 años de actividad ordenada y sistematizada, que conjuga conservación, manejo silvícola y producción maderera (1000 a $3000 \mathrm{~m}^{3}$ /año), investigación (Attis Beltrán et al. 2015, 2018; Chauchard and Sbrancia 2003; Chauchard et al. 2012, 2016; Dezzoti et al. 2014; Sola et al. 2015; Sola 2016), educación (Tecnicatura Forestal del AUSMA), y todo bajo la particularidad institucional y normativa de la Administración de Parques Nacionales. El análisis central que se debería realizar en este caso tiene dos aspectos de ejemplaridad. Uno es que el Estado (en la figura de la Administración de Parques Nacionales) promociona, guía y monitorea: el manejo forestal a través de planes sistematizados en campos privados y públicos de la Reserva Nacional Lanín, que han tenido un seguimiento atento. Este posicionamiento permitió llevar adelante el concepto de manejo forestal adaptativo, que pudo contribuir a un desarrollo forestal local, ambiental, económica y socialmente aceptable y beneficioso. El otro aspecto lo constituye el foco de cuenca o paisaje que tuvo la planificación de los aprovechamientos.
Este soporte permitió trascender el manejo de un propietario particular para llevarlo a un proceso con base sustentable a nivel de cuenca que ha contenido los numerosos planes de manejo implementados en estas tres décadas. Todo esto fue favorecido por la institucionalidad, que promovió un manejo que integra la ecología, la rentabilidad, los intereses de los propietarios, las empresas de servicios, las inversiones, las industrias, el mercado local y, finalmente, la sociedad, que recibe una variedad de productos madereros. Vale resaltar que una parte ejemplar de los manejos forestales en el Parque Nacional Lanín lo determina que, además del seguimiento anual, en tierras fiscales o de propietarios, todos los planes tienen sus revisiones ordinarias a los 5 ó 10 años. En la Argentina, al igual que en Latinoamérica, se pueden encontrar casos similares de empresas o de propietarios que trabajan los bosques con altos estándares de responsabilidad ambiental y social. Cuando ello ocurre en un escenario en el cual es frecuente el deterioro del ambiente, con falta de planificación orientada al desarrollo del sector a escala de paisaje o cuenca, se denomina a estos casos ejemplares como envasados o enlatados. Por un lado, esto sucede por decisiones propias de particulares o de empresas, que deciden llevar adelante un manejo forestal sustentable y, por el otro lado, porque generalmente el Estado está ausente para fomentar la repetición del éxito en el resto de la región.

Los productores no suelen tener acceso a un conocimiento técnico que le permita optimizar sus prácticas y su economía, y siguen usando el bosque de una manera tradicional de base extractiva, muchas veces asociada a usos ganaderos, en un proceso sin planificación integral espacial y temporal. A menudo, esto resulta en procesos de degradación. La falta de acceso al conocimiento técnico por parte de los productores, combinada con la falta de diálogo efectivo entre los sectores académicos, institucional y productivo, es uno de los determinantes de la ausencia de manejo de gran parte de los bosques de la región. Y cuando están bajo un aprovechamiento, éste suele estar disociado de los criterios sugeridos por la academia. Tampoco contribuyeron a cambiar la realidad forestal las escasas iniciativas de una extensión forestal efectiva por parte de las instituciones de ciencia y técnica de la región, así como la falta de aplicación de la legislación vigente y la deficiencia en los mecanismos de control por parte del Estado. 
No es sencillo generar un diálogo abierto entre funcionarios, académicos, ONG ambientales, propietarios, ocupantes y comunidades de origen muy diverso. Es común confiar en quien tiene una formación social y cultural similar a la nuestra y desconfiar del resto. En el caso del bosque, las diferencias sociales, económicas y culturales entre académicos, funcionarios públicosy representantes deONG - quienes provienen de una sociedad urbana y transitaron por un sistema de aprendizaje teórico y general- y los productores, pobladores y comunidades rurales — quienes basan gran parte de su aprendizaje en el conocimiento práctico y local-generan, en muchos casos, desconfianza mutua y actitudes de exclusión o autoexclusión. La conformación de Comisiones Consultivas de la Ley de Bosques Nativos representa una buena propuesta para el dialogo intersectorial, pero que hasta ahora fracasó por falta de convocatoria y continuidad por parte de las Autoridades de Aplicación y de hechos concretos que estimulen a los productores a seguir participando.

Otra faz importante del problema lo constituye la carencia de políticas públicas explícitas y de acciones efectivas y sostenidas de los organismos del Estado, que constituyen la autoridad de aplicación en el sector. Claramente, la Ley de Bosques, su articulación con otras leyes como las de Promoción Forestal o aquellas vinculadas al empleo, la industria y la comercialización, deben ser instrumentos de lineamientos políticos explícitos, canalizados a definir una visión hacia el desarrollo y la planificación de manejo que partan de las escalas de paisaje o cuenca. La escala de planificación (i.e., provincial, regional, cuenca o local) y el o los regímenes de tenencia de la tierra (pública, privada, comunitaria, ocupación, comodato, etc.), asociada a dicha escala determinarán el grado de complejidad para definir objetivos de manejo, así como para determinar cuáles son los instrumentos (e.g., incentivos, infraestructura, mesas sectoriales, presencia territorial, etc.) necesarios para que los propietarios aborden el desafío de manejar sus bosques y poner la producción en el mercado bajo un esquema sustentable.

\section{¿No PODEMOS O NO DEJAMOS?}

Históricamente, a lo largo de los diferentes paradigmas forestales que se propusieron de forma explícita o implícita desde el sector público-académico (Tabla 1), los intereses, las expectativas y los deseos de los pobladores, comunidades y productores que viven y usan el bosque fueron desatendidos. Durante todo este proceso se desarrolló una relación antagónica entrelas Autoridades de Aplicación y la población rural local. Los organismos del Estado determinaron e impusieron usos habilitados y no habilitados, basados en una concepción extraña a la opinión y la cultura local, que incluso llevó en algunos casos a la expulsión o relocalización de los pobladores.

Las políticas de colonización, de conservación a ultranza, de explotación forestal basada sobre permisos anuales con escasa planificación territorial, de forestaciones con especies exóticas como mecanismo de compensación y apropiación territorial que resultaron perjudiciales para el bosque nativo fueron formuladas adaptando modelos provenientes de Europa y de Norteamérica. Sin embargo, no tuvieron en cuenta las características propias de estos ecosistemas y no contemplaron las necesidades y expectativas de las comunidades locales respecto del uso del territorio (ver Foro "Ecología y Sociedad" en Ecología Austral 25[2]: Agosto 2015). Como consecuencia de este proceso, hoy existe una desconfianza mutua, como se expuso anteriormente, entre pobladores y funcionarios públicos que se refleja en los conflictos en la aplicación de las normativas vigentes.

Resulta paradójico que 10 años después de su aprobación, la denominada "Ley de Bosques Nativos" - promovida desde sectores principalmenteurbanos y mayormentecon una visión y preocupación por la conservación y no por el uso productivo- no logró incentivar el manejo sustentable del recurso forestal (i.e., maderero y no maderero) por parte de los Estados provinciales y pobladores de nuestra región. Los pobladores no fueron informados de manera fehaciente ni consultados sobre las sanciones y los efectos de las normativas a nivel nacional y provincial que influyen sobre los bosques de las propiedades; actualmente, tienen una participación escasa en la toma de decisiones en relación con su propio espacio territorial.

A la fecha, más allá de los graves problemas de falta de financiación de esta ley, no existen en Patagonia Norte equipos técnicos capacitados y con recursos suficientes para asistir a pobladores y productores en el manejo sustentable de los bosques nativos. Tampoco hay grupos de extensión que faciliten y promuevan la incorporación de las técnicas 
Tabla 1. Evolución de los paradigmas forestales en Patagonia Norte.

Table 1. Evolution of forest paradigms in North Patagonia

\begin{tabular}{|c|c|c|c|}
\hline Período & Paradigma & Política pública & Resultados \\
\hline $1870-1930$ & $\begin{array}{l}\text { Colonización y expansión } \\
\text { agrícolo-ganadera }\end{array}$ & $\begin{array}{l}\text { Quemazones de bosques } \\
\text { para apertura de pasturas. }\end{array}$ & Pérdida de cobertura forestal \\
\hline 1930-1970 & $\begin{array}{l}\text { Consolidación territorial e } \\
\text { Industrialización }\end{array}$ & $\begin{array}{l}\text { Creación de los parques } \\
\text { nacionales y fomento de } \\
\text { grandes aserraderos de } \\
\text { especies nativas. }\end{array}$ & $\begin{array}{l}\text { Grandes superficies boscosas } \\
\text { protegidas. Creación de } \\
\text { poblados asociados localmente } \\
\text { a concesiones madereras de } \\
\text { explotación intensiva. }\end{array}$ \\
\hline 1970-2007 & $\begin{array}{l}\text { Desarrollo y concentración } \\
\text { económica }\end{array}$ & $\begin{array}{l}\text { Promoción de } \\
\text { forestaciones exóticas } \\
\text { de rápido crecimiento. } \\
\text { Abandono de la } \\
\text { explotación del BN con } \\
\text { fines comerciales. }\end{array}$ & $\begin{array}{l}\text { Plantaciones diseminadas en } \\
\text { el territorio en varias cuencas. } \\
\text { Muchas forestaciones no fueron } \\
\text { manejadas, lo que dio comienzo } \\
\text { al proceso de invasión del BN } \\
\text { por especies exóticas. }\end{array}$ \\
\hline 2007-actualidad & Sustentabilidad & $\begin{array}{l}\text { Ley de presupuestos } \\
\text { mínimos para la } \\
\text { protección ambiental de } \\
\text { los bosques nativos. }\end{array}$ & $\begin{array}{l}\text { Aún no es posible evaluar sus } \\
\text { resultados, pero en } 10 \text { años los } \\
\text { avances en el manejo forestal } \\
\text { son muy escasos. }\end{array}$ \\
\hline
\end{tabular}

silvícolas desarrolladas desde la ciencia y la técnica. Por otra parte, las Administraciones establecieron circuitos administrativos engorrosos y disociados de la realidad de los productores rurales, quienes ven inhibidas todas sus intenciones de iniciar el manejo de sus bosques (Gowda 2010a, 2010b). Prueba de ello es que en esta región aún no se aprobaron planes de manejo productivo de bosques nativos, lo que genera desconfianza por parte de los pobladores y desalienta su participación futura y el acogimiento a normas legales.

En la Patagonia Norte existe una marcada disociación entre el valor de la tierra y su valor productivo; esto se relaciona con el valor escénico, la distancia a las ciudades y los cursos de agua, y con su valor turístico e inmobiliario. Más allá de eso, existe un mercado instalado que premia las especies nativas por sobre las implantadas. Las especies más valoradas en la región son el ciprés, el raulí, el roble pellín, la lenga y la araucaria. Del ciprés sólo se permite, hasta ahora, la extracción de individuos muertos de baja calidad maderera, pero con usos importantes en construcción como vigas y columnas; el raulí y el roble pellín que se extraen del PN Lanín se utilizan con diversos fines en construcción y carpintería, mientras que la lenga y la araucaria, consideradas de muy alta calidad, no son explotadas por restricciones legales (salvo la lenga en Chubut). Consideramos que existe un enorme mercado potencial si el Estado aprueba los planes de manejo presentados, tanto para su industrialización como para su uso energético.

\section{¿SEguimos PARTICIPANDO DE LA} TEORÍA O EMPEZAMOS A HACER ALGO?

Más allá de los procesos históricos y los conflictos que generaron entre distintos actores con diferentes grados de responsabilidad sobre el manejo sustentable del bosque, la aprobación de la Ley 26331 constituye un instrumento concreto para la planificación e implementación de políticas públicas. Bajo el concepto de manejo sustentable incluimos la articulación entre la conservación, la producción y la restauración del bosque nativo.

Dado que, en la práctica, el manejo de los bosques será realizado por los productores locales — quienes asumen ante la ley la responsabilidad directa de los resultados del manejo-, el primer paso para un manejo sustentable es considerar el uso actual, sus necesidades y expectativas. Muchos productores provienen de una cultura tradicionalmente agrícola-ganadera y no consideran al bosque nativo como parte de su desarrollo productivo. Por un lado, esto sucede porque en las provincias patagónicas, el Estado considera al bosque como un recurso propio; por otro lado, debido a que el Estado no es capaz de ordenar las actividades productivas que se llevan o se pueden llevar a cabo en él.

Un primer avance para incentivar la restauración y el manejo productivo de los bosques, presente y futuro, sería asociar explícitamente en los planes el manejo 
adaptativo. Éste deberá integrar todos los usos actuales del bosque y el derecho al uso efectivo del recurso forestal a quienes son responsables directos de su manejo.

En segundo lugar, es fundamental que desde los organismos del Estado se considere a los productores como interlocutores indispensables a la hora de generar normativas e, inclusive, para trazar una visión para la región, ya que los mecanismos participativos suelen estar ausentes o no son suficientemente abiertos. Esto implica no sólo involucrarlos desde el comienzo en los procesos de planificación a distintas escalas, sino también acompañarlos con una presencia concreta en el territorio, con técnicos y extensionistas bien capacitados para asistir a las demandas que el manejo acordado requiera.

En tercer lugar, el sistema administrativo se debe adecuar a lograr eficacia de la gestión y eficiencia en el tiempo de los trámites, y debe considerar, fundamentalmente, la realidad del productor. Ejemplos puntuales son: a) los tiempos administrativos largos que no condicen con los tiempos productivos [como ejemplo podemos citar lo que sucede con los Planes Operativos Anuales (POA), que se aprueban en plazos demasiado largos de meses a años, cuando deberían resolverse en períodos menores a 90 días. La división entre aprobación técnica y financiera de los Planes de Manejo y sus respectivos POA sería un mecanismo sencillo para facilitar este proceso de adecuación], b) la presencia frecuente de técnicos con cierto poder de decisión a escala predial en las zonas de producción reduciría la distancia actual entre productores o concesionarios y funcionarios e instituciones públicas, y con ello se agilizarían los tiempos para casos como consultas o solución de problemas de campo. Es usual que los técnicos estén radicados en áreas urbanas, distanciándose no solo de los productores rurales, como se expuso, sino también de la posibilidad de tener un control y monitoreo más eficaz del proceso, disminuyendo también los costos y tiempos de traslados, en especial de los productores rurales.

De forma complementaria, el conocimiento empírico de los productores locales que hoy realizan actividades en los bosques nativos debe ser valorado adecuadamente, tanto por la academia como por los tomadores de decisiones. En la mayoría de los centros universitarios de formación profesional, tanto de orientación forestal como ambiental, se deberían generar más ámbitos de interacción para aprender y orientar las investigaciones asociadas al manejo productivo de los bosques nativos desde la discusión para una co-construcción del conocimiento que guíe un aprendizaje adaptativo entre los diferentes actores del sector.

Por último, las prácticas experimentales exitosas se deberían poder adecuar a una escala productiva, tanto a nivel predial como de paisaje o de cuenca, y abordar problemas de la ordenación del uso múltiple a esta escala supra-predial con la que se pueda ofrecer una alternativa a la producción forestal para la cuenca para el mediano plazo en una visión de largo plazo, involucrando a todos los tenedores de bosques de la región de análisis. En este contexto, se propone que las autoridades de aplicación establezcan una "red para la gestión" con instituciones como el INTA, el CIEFAP, el CONICET y las universidades, entre otras. Con la responsabilidad ineludible de promover planes adaptativos (flexibles) que permitan aprender y mejorar prácticas a largo plazo, con un monitoreo permanente y con ello tratar de resolver qué acordamos para definir el manejo forestal sustentable en términos de gestión.

Concluimos que en la Patagonia Norte existen los conocimientos técnicos suficientes para iniciar un proceso de manejo forestal sustentable. Sin embargo, faltaría una clara voluntad por parte de los organismos de gestión, investigadores y profesionales del sector para promover el uso del bosque nativo.

\section{REFERENCIAS}

Attis Beltrán, H., L. Chauchard, and G. Martínez Pastur. 2015. Curvas preliminares de índice de sitio para bosques puros y mixtos de Nothofagus alpina y Nothofagus obliqua en la Patagonia argentina. Bosque 36(2):275-285.

Attis Beltrán, H. 2016. Relaciones entre la estructura forestal y el rendimiento del bosque de Nothofagus alpina y Nothofagus obliqua en gradientes de edad y calidad de sitio". Tesis doctoral, Universidad Nacional del Comahue, Río Negro, Argentina. Pp. 235.

Bava, J. 1999. Los bosques de lenga en la Argentina. Pp. 273-279 en C. Donoso and A. Lara (eds.). Silvicultura de los bosques nativos de Chile. Universidad Austral. Ed. Universitaria, Valdivia, Chile.

Bava, J., and J. P. López Bernal. 2006. Las cortas de selección en grupo en bosques de lenga de Tierra del Fuego. Quebracho 13:87-82. 
Blackhall, M., E. Raffaele, J. Paritsis, F. Tiribelli, J. Morales, T. Kitzberger, J. H. Gowda, and T. T. Veblen. 2017. Effects of biological legacies and herbivory on fuels and flammability traits: A longdterm experimental study of alternative stable states. Journal of Ecology 105(5):1309-1322.

Chauchard, L., and R. Sbrancia. 2003. Modelos de crecimiento diamétrico para Nothofagus obliqua. Bosque 24(3):3-16

Chauchard, L., J. Bava, S. Castañeda, P. Laclau, G. Loguercio, P. Pantaenius, and V. Rusch. 2012. Manual para las buenas prácticas forestales en bosques nativos de norpatagonia. Unidad para el Cambio Rural, Ministerio de Agricultura, Ganadería y Pesca, Buenos Aires, Argentina.

Chauchard, L., M. C. Frugoni, and C. Novak. 2015. Manual de Buenas Prácticas para el Manejo de las Plantaciones Forestales en la Región de la Patagonia Andina. Unidad para el Cambio Rural, Ministerio de Agricultura, Ganadería y Pesca, Buenos Aires, Argentina.

Dezzotti, A., R. Sbrancia, D. Roat, M. Rodríguez-Arias, and A. Parisi. 2004. Colonización y crecimiento de renovales de Nothofagus después de cortas selectivas de un rodal en la Patagonia, Argentina. Investigación Agraria Serie Forestal 13(2):329-337.

Goldenberg, M., J. H. Gowda, C. Casas, and L. A. Garibaldi. 2018. Efecto de la tasa de descuento sobre la priorización de alternativas de manejo del matorral Norpatagónico argentino. Bosque 39(2):217-226.

Gowda, J. H. 2010a. La ilegalidad institucionalizada. Principal enemigo de nuestros bosques. Cap. I. Noti Forestal 217:5-7.

Gowda, J. H. 2010b. La ilegalidad institucionalizada, principal enemiga de nuestros bosques. Cap II. Noti Forestal 217:9-14.

Gowda, J. H., T. Kitzberger, and A. C. Premoli. 2012. Landscape responses to a century of land use along the northern Patagonian forest-steppe transition. Plant Ecology 213(2):259-272.

Gowda, J. H., T. Kitzberger, and L. Suárez. 2014. Comunidades vegetales y ecosistemas terrestres. Informe final. Parque nacional Nahuel Huapi, Bariloche, Río Negro, Argentina.

Kitzberger, T., G. L. Perry, J. Paritsis, J. H. Gowda, A. J. Tepley, A. Holz, and T. T. Veblen. 2016. Fire-vegetation feedbacks and alternative states: common mechanisms of temperate forest vulnerability to fire in southern South America and New Zealand. New Zealand Journal of Botany 54(2):247-272.

Kitzberger, T., and T. T. Veblen. 1999. Fire-induced changes in northern Patagonian landscapes. Landscape Ecology 14(1):1-15.

Mundo, I. A., T. Kitzberger, F. A. Roig Juñent, R. Villalba, and M. D. Barrera. 2013. Fire history in the Araucaria araucana forests of Argentina: Human and climate influences. International Journal of Wildland Fire 22(2):194-206.

Sola, G. 2016. Impacto del manejo silvícola sobre la estructura demográfica y genética de la regeneración natural en el bosque mixto de Nothofagus. Tesis doctoral. Universidad Nacional del Comahue, Bariloche, Río Negro, Argentina.

Sola, G., H. Attis Beltrán, L. Chauchard, and L. Gallo. 2015. Efecto del manejo silvicultural sobre la regeneración de un bosque de Nothofagus dombeyi, N. alpina y N. obliqua en la Reserva Nacional Lanín (Argentina). Bosque 36(1): 113-120.

Raffaele, E., T. T. Veblen, M. Blackhall, and N. Tercero-Bucardo. 2011. Synergistic influences of introduced herbivores and fire on vegetation change in northern Patagonia, Argentina. Journal of Vegetation Science 22:59-71.

Rusch, V., and M. Sarasola. 1999. Empleo de Criterios e Indicadores en el manejo Forestal Sustentable. Biodiversidad. Parte I, Propuesta metodológica. Pp. 15-24 en Actas Segundas Jornadas Iberoamericanas sobre Diversidad Biológica, Tomo II. San Luis, Argentina.

Tercero-Bucardo, N., T. Kitzberger, T. T. Veblen, and E. Raffaele. 2007. Afield experiment on climatic and herbivore impacts on post-fire tree regeneration in north-western Patagonia. Journal of Ecology 95:771-779.

Urretavizcaya, M. F. 2006. Ciprés de la cordillera, Plantación de bosques quemados y recomendaciones para su establecimiento. Patagonia Forestal 12(4):13-16.

Willis, B. 1914. El Norte de la Patagonia, naturaleza y riquezas. Scribner Press. New York, USA. 